\section{MS42-05 Stroboscopic neutron powder diffraction at HRPT, SINQ}

Denis Sheptyakov ${ }^{1}$, Vladimir Pomjakushin ${ }^{1}$, Lucien Boulet-Roblin $^{2}$, Claire Villevieille ${ }^{2}$, Gerd Theidel ${ }^{3}$, Mark Könnecke ${ }^{4}$, Roman Bürge ${ }^{4}$

1. Laboratory for Neutron Scattering and Imaging, Paul Scherrer Institute, 5232 Villigen PSI, Switzerland

2. Electrochemistry Laboratory, Paul Scherrer Institute, 5232 Villigen PSI, Switzerland

3. Laboratory for Particle Physics, Paul Scherrer Institute, 5232 Villigen PSI, Switzerland

4. Laboratory for Scientific Developments and Novel Materials, Paul Scherrer Institute, 5232 Villigen PSI, Switzerland

email: denis.cheptiakov@psi.ch

At HRPT, the high-resolution powder neutron diffractometer at SINQ*, a new mode of operation has been realized and tested. This stroboscopic measurements mode is intended for studies of crystal or magnetic structure transformations occurring in short (available time binning down to 10 milliseconds), but multiply repeating processes triggered by some external effect, e.g. voltage, magnetic field, thermal heat pulse, etc. The diffraction data corresponding to different time slices after a certain repeating strobo signal are stored in the different histogram memory blocks (up to about 70000 independent diffraction patterns). In our tests, we could successfully slice time down to 10 milliseconds with overall duration of experiment of several hours, so the processes, or existence ranges of phases on the order of tens of milliseconds, seconds, minutes etc., - can now be addressed efficiently by neutron diffraction at HRPT. The first real studies, including the operando investigation of ultrafast charging-discharging cycles of a commercial Li-ion battery ( $\mathrm{LiFePO}_{4}$ vs. graphite) will be presented.

* https://www.psi.ch/sinq/hrpt/hrpt

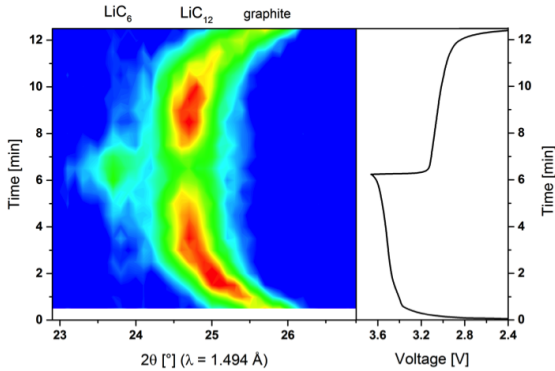

Figure 1. Selected sections of the stroboscopic neutron powder diffraction patterns corresponding to 120 charge-discharge cycles of a battery, showing transition between graphite, $\mathrm{LiC}_{12}$ and $\mathrm{LiC}$ phases and back. Overall charge-discharge time is approximately 12 min. Data binned with 30 seconds per pattern.

Keywords: stroboscopic, neutron diffraction
MS43 Combining x-ray

diffraction and other techniques

for in situ and operando studies

Chairs: Helmut Ehrenberg, François Fauth

MS43-01 Revealing Secrets of Lithium-Ion

Battery Operation by Neutron Scattering

Martin J. Muehlbauer ${ }^{1,2,3}$, Anatoliy Senyshyn ${ }^{3}$, Michael Knapp ${ }^{1,2}$, Helmut Ehrenberg ${ }^{1,2}$

1. Helmholtz-Institute Ulm for Electrochemical Energy Storage (HIU), P.O. Box 3640, D-76021 Karlsruhe, Germany

2. Institute for Applied Materials (IAM), Karlsruhe Institute of Technology (KIT), Hermann-von-Helmholtz- Platz 1, D-76344 Eggenstein-Leopoldshafen, Germany

3. Heinz Maier-Leibnitz Zentrum (MLZ), Technische Universität München, Lichtenbergstr. 1, 85748 Garching, Germany

email: martin.muehlbauer@kit.edu

Powering innumerable portable devices lithium-ion batteries are part of our everyday life for decades already. Although lithium-ion chemistry does a good job powering consumer electronics and tools, there are still some processes inside lithium-ion batteries that are not understood completely. Especially an increasing number of applications related to electromobility and energy storage calls for further improvements of their life span, energy/power density and rate capability. Therefore, single cells or even integrated batteries have to be investigated under real operating conditions to unravel details occurring in the millimetre to micrometre domain and reaching down to a nanometre or even atomic length scale. Neutrons offer a capability to conduct in operando investigations on standard size Li-ion cells even in combination with additional sample environment, e.g. to study temperature effects. For low temperatures an intercalation behaviour differing from the one at room temperature has been observed inside the graphitic anode [1]. Neutron radiation is not only sensitive for light elements but also enables to distinguish neighbouring elements. Therefore, structural changes, phase transitions and cation exchange reactions, e.g. in cathode materials, may be traced during intercalation and deintercalation of lithium providing detailed information about battery operation [2]. Inhomogeneities of the state of charge inside 18650-type Li-Ion cells were pointed out by neutron imaging and neutron diffraction experiments. They could be quantified by spatially resolved neutron diffraction on a macroscopic length scale as inhomogeneities of the lithium concentration inside the anode for fully charged cells [3]. Here a correlation between the cell design and the lithiation state of the graphitic anode has been found.

Results based on electrochemical cell characterization, neutron imaging and scattering techniques will be presented. They will be discussed in terms of their 
influence for the design and operation of Li-ion cells, i.e. cell geometry (tab positions), cell balancing or operating temperature.

[1] A. Senyshyn, M.J. Mühlbauer, O. Dolotko, H. Ehrenberg., Journal of Power Sources 282 235-240 (2015).

[2] O. Dolotko, A. Senyshyn, M.J. Mühlbauer, K. Nikolowski, H. Ehrenberg, Journal of Power Sources, 245 197-203 (2014).

[3] A. Senyshyn, M.J. Mühlbauer, O. Dolotko, M. Hofmann, H. Ehrenberg, Nature Scientific Reports, 5, 18380 (2015), doi: 10.1038/srep18380.
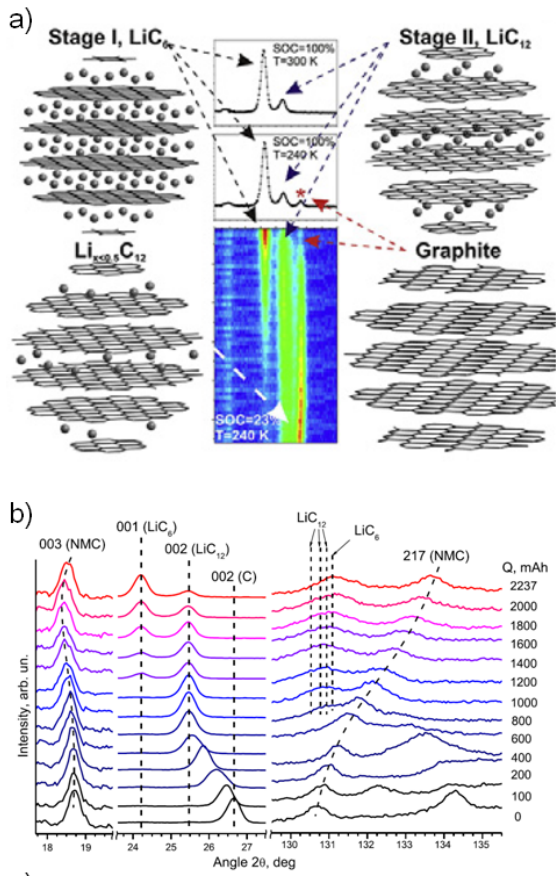

c)

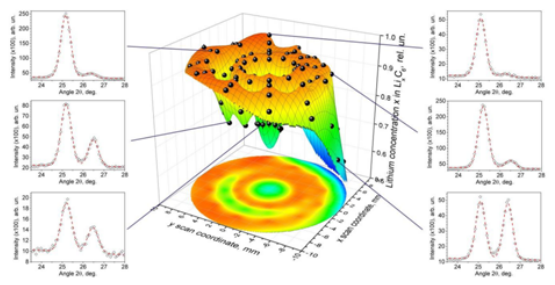

Figure 1. a) In operando neutron diffraction during discharge at $240 \mathrm{~K}$, intercalation behaviour differs from room temperature [1]; b) diffraction data at different state of charge point out cation mixing in NMC cell [2]; c) spatially resolved lithium concentration inside the anode of a charged $\mathrm{Li}$-ion cell [3]
MS43-O2 New insight on structural and redox processes involved upon cycling of $\mathrm{Na}_{3} \mathrm{~V}_{2}\left(\mathrm{PO}_{4}\right)_{2} \mathrm{~F}_{3}$, an attractive positive electrode material for $\mathrm{Na}$-ion batteries

Thibault Broux ${ }^{1,2,3}$, Bianchini Matteo ${ }^{1,2,4}$, Fauth François ${ }^{5}$, Simonelli Laura ${ }^{5}$, Stievano Lorenzo ${ }^{3,6}$, Suard Emmanuelle ${ }^{4}$, Masquelier Christian ${ }^{2,3}$, Croguennec Laurence ${ }^{1,3}$

1. CNRS, University of Bordeaux, Bordeaux INP, ICMCB UPR 9048, F-33600 Pessac, France

2. LRCS, CNRS-UMR 7314, University Picardie Jules Verne, F-80039 Amiens Cedex 1, France

3. RS2E, FR CNRS 3459, F-80039 Amiens Cedex 1, France

4. Institut Laue-Langevin, F-38000 Grenoble, France

5. CELLS - ALBA synchrotron, E-08290 Cerdanyola del Vallès, Barcelona, Spain

6. ICG, CNRS-UMR 5253, University of Montpellier, F-34095 Montpellier Cedex 5, France

email: Thibault.Broux@icmcb.cnrs.fr

As electrode materials for batteries are operating in non-equilibrium conditions a deeper understanding of the structural and redox processes occurring upon cycling can be achieved using operando techniques. In order to do this a versatile electrochemical cell has been developed for X-ray experiments allowing real-time data collection upon charge/discharge (i.e. upon lithium or sodium extraction/insertion from/into the electrodes), either by lab X-ray and synchrotron X-ray powder diffraction or by $\mathrm{X}$-ray absorption spectroscopy [1]. This unveils dynamics features that are not accessible by other means and gives a greater picture of the electrodes' functioning from electronic and atomic point of view.

Concerning $\mathrm{Na}_{3} \mathrm{~V}_{2}\left(\mathrm{PO}_{4}\right)_{2} \mathrm{~F}_{3}$ recent structural characterization of the fully charged material $\mathrm{Na}_{1} \mathrm{~V}_{2}\left(\mathrm{PO}_{4}\right)_{2} \mathrm{~F}_{3}$ via operando synchrotron X-ray diffraction performed at the ALBA synchrotron (Barcelona, Spain) suggests charge disproportionation of $2 \mathrm{~V}(+\mathrm{IV})$ into $\mathrm{V}(+\mathrm{III})$ and $\mathrm{V}(+\mathrm{V})$. Indeed structural features combined with bond valence sums show two different vanadium environments consistent with such a hypothesis [2]. To further support this observation operando X-ray absorption near edge spectroscopy (XANES) at the vanadium K-edge in fluorescence mode has been performed at ALBA which gives relevant information on local environment and electronic configuration of the vanadium, especially through the pre-edge peaks' investigation. The obtained data have been analyzed via principal component analysis and multivariate curve resolution which constitute a novel approach to characterize electrode materials in operating conditions.

Furthermore impact of the cycling rate and temperature on the phase diagram observed upon sodium deintercalation and re-intercalation from/in $\mathrm{Na}_{3} \mathrm{~V}_{2}\left(\mathrm{PO}_{4}\right)_{2} \mathrm{~F}_{3}$ will be also extensively discussed.

1. Leriche, J. B.; Hamelet, S.; Shu, J.; Morcrette, M.; Masquelier, C.; Ouvrard, G.; Zerrouki, M.; Soudan, P.; Belin, S.; Elkaïm, E. and Baudelet, F., J. Electrochem. Soc. 2010, 157, A606-A610.

2. Bianchini, M.; Fauth, F.; Brisset, N.; Weill, F.; Suard, E.; Masquelier, C. and Croguennec, L., Chem. Mater. 2015, 27(8), 3009-3020. 